\title{
Genetic Algorithm Based Antenna Diversity Assisted Multiuser Detection for Synchronous CDMA Systems
}

\author{
Kai Yen and Lajos Hanzo \\ Dept. of ECS, Univ. of Southampton, SO17 1BJ, UK. \\ Tel: +44-703-593 125; Fax: +44-703-594 508 \\ Email: lh@ecs.soton.ac.uk; http://www-mobile.ecs.soton.ac.uk
}

\begin{abstract}
A spatial diversity reception assisted multiuser CDMA detector based on genetic algorithms (GAs) is proposed. Two different diversity selection strategies are considered. In our first approach the so-called individuals of the GA are chosen for selection based purely on the sum of their corresponding figures of merit associated with the individual antennas, an approach which is analogous to employing the conventional log-likelihood function (LLF) for diversity reception. According to our second strategy, the individuals are chosen for selection based on the concept of the so-called Pareto optimality, which uses the information from the individual antennas independently. Computer simulations showed that the GAs employing the latter strategy achieve a lower BER as compared to the former approach.
\end{abstract}

\section{INTRODUCTION}

It is well known that the multiple access interference (MAI) present in code division multiple access (CDMA) [1, 2] systems can seriously deteriorate the quality of reception. An attractive solution for eliminating or reducing the effects of MAI is multiuser detection [3]. The optimum multiuser detector [4] - which is based on the maximum likelihood (ML) rule in the context of synchronous CDMA systems - searches exhaustively for the specific sequence of the users' transmitted bits that maximises the so-called log-likelihood function (LLF). Since the number of possible bit sequence combinations is exponentially proportional to the number of users, the optimum multiuser detector has a computational complexity that is exponentially increasing with the number of users. Hence it is impractical to implement. This limitation led to numerous so-called suboptimal multiuser detection proposals, highlighted in [5] and in the references therein, which sacrifice performance for the sake of a reduced complexity.

Genetic algorithms (GAs) [6,7] have been employed for solving many complex optimization problems in numerous fields. While GAs are by no means perfect, i.e. they do not always find the optimal point in the optimization space, they are very efficient in attaining near-optimal solu-

This work has been performed in the framework of the PanEuroepan IST project IST-1999-12070 (TRUST), which is partly funded by the European Union. The authors would like to acknowledge the contributions of their colleagues, although the views expressed are those of the authors.

The financial support of the EPSRC, Swindon, UK is also gratefully acknowledged.

VTC2001 Spring, Rhodes, Greece tions significantly faster, than conventional point-by-point exhaustive search techniques, especially in large solution spaces. GA-based multiuser detection has been first proposed in $[8,9]$, where the analysis was based on the AWGN channel without invoking diversity techniques. In [10], Rayleigh fading channels were considered, again, in the absence of diversity techniques.

In this contribution, we present a novel approach to the problem of multiuser detection in DS/CDMA over flatfading channels assisted by antenna diversity [11] based on a genetic algorithm innovation. The antennas are assumed to be sufficiently separated such that the received signals at the antennas are faded independently, resulting in an independent LLF for each antenna. This imposes a problem on the optimization process due to the fact that while a specific bit sequence may optimize the LLF of one antenna, the same bit sequence may not necessarily optimize the LLF of the other antenna. In order to resolve this dilemma two different GA-based diversity selection strategies are considered. In our first approach, the individuals of the GA are chosen for selection based purely on the sum of their corresponding figures of merit from the two antennas, an approach which is analogous to invoking the conventional LLF for diversity reception [12]. According to our second strategy, the individuals associated with the GA are chosen for selection based on the concept of the so-called Pareto optimality [6], which uses the information from the antennas independently.

This paper is organized as follows. Section II describes our synchronous CDMA system communicating over uncorrelated non-frequency-selective fading channels using two antennas. Section III describes the GAs used for implementing our proposed detector in conjunction with diversity reception. Our simulation results are presented in Section IV, while Section V concludes the paper.

\section{System Description}

We consider a symbol-synchronous CDMA system, where $K$ users are simultaneously transmitting binary phase shift keying (BPSK) modulated bits having a period $T_{b}$. Each bit is spread by a user-specific signature sequence having a chip period $T_{c}$, hence the processing gain of the system becomes $N_{c}=T_{b} / T_{c}$.

The receiver shown in Fig. 1 consists of two antennas 


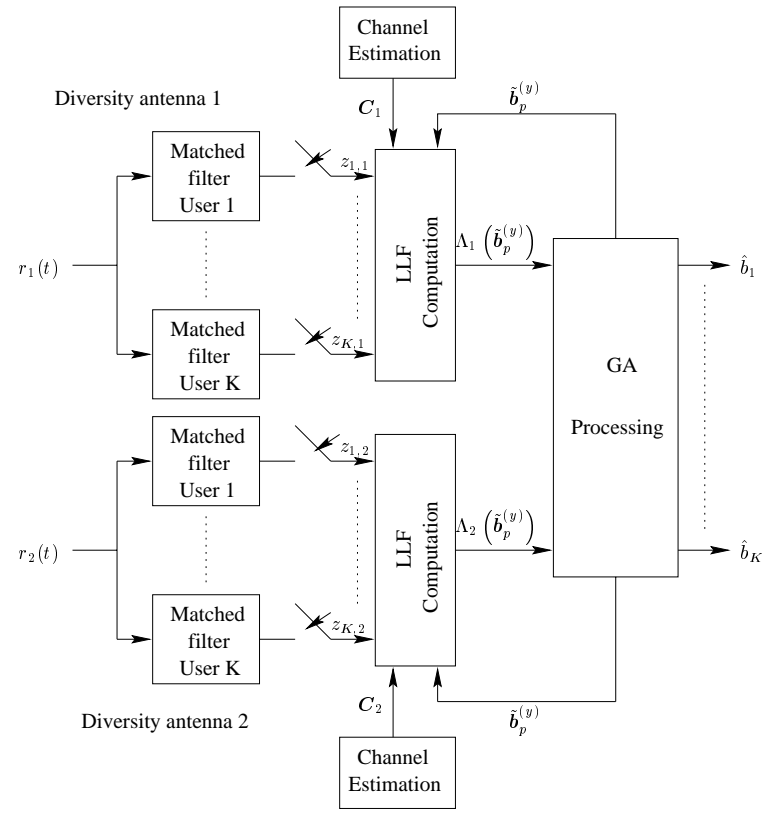

Fig. 1. Block diagram of the receiver model

separated spatially, such that the signals received are statistically independent. Asumming ideal lowpass filtering, the baseband received signal at the $i$ th antenna is given by :

$$
r_{i}(t)=\boldsymbol{a}^{T}(t) \boldsymbol{\xi} \boldsymbol{C}_{i} \boldsymbol{b}+n_{i}(t), \quad 0 \leq t<T_{b}
$$

where $\boldsymbol{a}(t)=\left[a_{1}(t) a_{2}(t) \ldots a_{K}(t)\right]^{T}$ is the signature sequence vector for the $K$ users, $\boldsymbol{\xi}=$ $\operatorname{diag}\left(\sqrt{\xi_{1}} \sqrt{\xi_{2}} \ldots \sqrt{\xi_{K}}\right)$ is the received bit energy matrix of the $K$ users during the bit-interval considered, $\boldsymbol{C}_{i}=$ $\operatorname{diag}\left(\alpha_{1, i} e^{j \theta_{1, i}} \alpha_{2, i} e^{j \theta_{2, i}} \ldots \alpha_{K, i} e^{j \theta_{K, i}}\right)$ is the CIR matrix describing a frequency-nonselective slowly Rayleigh fading channel of each of the $K$ users, $\boldsymbol{b}=\left[b_{1} b_{2} \ldots b_{K}\right]^{T}$ is the transmitted bit vector of the $K$ users in the bit interval concerned and $n_{i}(t)$ is the zero-mean complex Additive White Gaussian Noise (AWGN) with independent real and imaginary components, each having a double-sided power spectral density of $N_{0} / 2$. The average received bit energyto-noise ratio of the $k$ th user is given by :

$$
\bar{\gamma}_{k}=\left\{E\left[\alpha_{k, 1}^{2}\right]+E\left[\alpha_{k, 2}^{2}\right]\right\} \gamma_{k},
$$

where $\gamma_{k}=\xi_{k} / N_{0}$ is the received bit signal-to-noise ratio (SNR) in the absence of fading. The path amplitudes are normalized, such that $E\left[\alpha_{k, 1}^{2}\right]+E\left[\alpha_{k, 2}^{2}\right]=1$ for $k=$ $1,2, \ldots, K$.

At each antenna, a bank of filters matched to the corresponding set of the users' signature sequences is sampled at the end of each bit interval. Hence, the output $\boldsymbol{z}_{i}$ of the matched filter bank at the $i$ th diversity antenna is given by the vector :

$$
\boldsymbol{z}_{i}=\left[z_{1, i} z_{2, i} \ldots z_{K, i}\right]^{T}=\boldsymbol{R} \boldsymbol{\xi} \boldsymbol{C}_{i} \boldsymbol{b}+\boldsymbol{n}_{i},
$$

where $\boldsymbol{R}=\int_{0}^{T_{b}} \boldsymbol{a}(t) \boldsymbol{a}^{T}(t)$ is a $K \times K$ user signature sequence cross-correlation matrix.
Based on the observation vector $\boldsymbol{z}_{i}$ given in Eq. (3), it can be shown that the LLF for the $i$ th antenna is given by [13] :

$$
\Lambda_{i}(\boldsymbol{b})=2 \Re\left\{\boldsymbol{b}^{T} \boldsymbol{C}_{i}^{*} \boldsymbol{\xi} \boldsymbol{z}\right\}-\boldsymbol{b}^{T} \boldsymbol{C}_{i} \boldsymbol{\xi} \boldsymbol{R} \boldsymbol{\xi} \boldsymbol{C}_{i}^{*} \boldsymbol{b} ; \text { for } i=1,2 .
$$

The decision rule for the optimum multiuser detector associated with the $i$ th antenna is to choose the bit vector $\hat{\boldsymbol{b}}$, which maximises the LLF given in Eq. (4). Hence, the estimated transmitted bit vector of the $K$ users is given by :

$$
\hat{\boldsymbol{b}}=\arg \left\{\max _{\boldsymbol{b}}\left[\Lambda_{i}(\boldsymbol{b})\right]\right\} .
$$

Since the channel characteristics for each antenna are statistically independent, we have typically $\Lambda_{1}(\boldsymbol{b}) \neq \Lambda_{2}(\boldsymbol{b})$ for the LLFs of the two antennae. In certain scenarios such as during deep fades, the above inequality implies that :

$$
\arg \left\{\max _{\boldsymbol{b}}\left[\Lambda_{1}(\boldsymbol{b})\right]\right\}=\hat{\boldsymbol{b}} \neq \arg \left\{\max _{\boldsymbol{b}}\left[\Lambda_{2}(\boldsymbol{b})\right]\right\}
$$

or vice versa. This creates a so-called multi-objective optimization problem, since the optimization of both LLFs can sometimes lead to two possible solutions. Note that in the conventional optimum detector [12], the LLFs corresponding to the two diversity antennas are summed in order to produce a scalar figure of merit, as given by [12] :

$$
\begin{aligned}
\Lambda(\boldsymbol{b}) & =\sum_{i=1}^{2} \Lambda_{i}(\boldsymbol{b}) \\
& =2 \Re\left\{\overrightarrow{\boldsymbol{b}}^{T} \overrightarrow{\boldsymbol{C}}^{*} \overrightarrow{\boldsymbol{\xi}} \vec{z}\right\}-\overrightarrow{\boldsymbol{b}}^{T} \overrightarrow{\boldsymbol{C}} \overrightarrow{\boldsymbol{\xi}} \overrightarrow{\boldsymbol{R}} \overrightarrow{\boldsymbol{\xi}} \vec{C}^{*} \overrightarrow{\boldsymbol{b}},
\end{aligned}
$$

where $\overrightarrow{\boldsymbol{b}}=\left[\begin{array}{lllll}b_{1} & b_{1} \ldots b_{K} & b_{K}\end{array}\right]^{T}$ and $\overrightarrow{\boldsymbol{z}}=\left[\begin{array}{lll}z_{1,1} z_{1,2} \ldots z_{K, 1} z_{K, 2}\end{array}\right]^{T}$ are vectors of dimension $2 K \times 1$, while $\overrightarrow{\boldsymbol{C}}=$ $\operatorname{diag}\left[\alpha_{1,1} e^{j \theta_{1,1}} \alpha_{1,2} e^{j \theta_{1,2}} \ldots \alpha_{K, 1} e^{j \theta_{K, 1}} \alpha_{K, 2} e^{j \theta_{K, 2}}\right]$ and $\overrightarrow{\boldsymbol{\xi}}=$ $\operatorname{diag}\left[\sqrt{\xi_{1}} \sqrt{\xi_{1}} \ldots \sqrt{\xi_{K}} \sqrt{\xi_{K}}\right]$ are diagonal matrices of dimension $2 K \times 2 K$. The decision rule is then to find the estimated transmitted bit vector $\hat{b}$ that maximizes $\Lambda(\boldsymbol{b})$.

In the next section we will highlight the philosophy of our GA-assisted diversity-aided multiuser detector with emphasis on the diversity selection strategy, in order to detect the users' transmitted bits.

\section{Genetic Algorithm Based Multiuser Detection with Diversity Reception}

GAs $[6,7]$ can be invoked in robust global search and optimization procedures that are well suited for solving complex optimization problems. In this contribution, we will employ GAs in order to detect the estimated transmitted bit vector $\hat{\boldsymbol{b}}$, where the so-called objective function is defined by the LLF of Eq. (4) for the two antennas.

GAs commence their search for the optimum solution at the so-called 0th generation by randomly creating $P$ number of sequences, each consisting of $K$ antipodal bits, where $P$ is known as the population size, which may assume values between 1 and $2^{K}$. These sequences are commonly referred to in GA terminology as individuals and each individual 
represents a possible solution of $\hat{\boldsymbol{b}}$. We shall express the $p$ th individual here as $\tilde{\boldsymbol{b}}_{p}^{(y)}=\left[\tilde{b}_{p, 1}^{(y)}, \tilde{b}_{p, 2}^{(y)}, \ldots, \tilde{b}_{p, K}^{(y)}\right]$, where $y$ denotes the $y$ th generation.

Associated with each individual we have two figures of merit, which are associated with the two antennas and are referred to as the so-called fitness values of the GA. These antenna-specific fitness values are derived by evaluating Eq. (4) with $\boldsymbol{b}$ as defined by the individual. The diversity-based fitness value of the $p$ th individual is denoted as $\boldsymbol{f}\left(\tilde{\boldsymbol{b}}_{p}^{(y)}\right)=\left[\Lambda_{1}\left(\tilde{\boldsymbol{b}}_{p}^{(y)}\right) \Lambda_{2}\left(\tilde{\boldsymbol{b}}_{p}^{(y)}\right)\right]$, which is a function of the LLFs associated with the two antennas. Based on the evaluated diversity-specific fitness, the population of $P$ individuals at the $y$ th generation of the GA evolves, in order to create a new population of $P$ individuals for the $(y+1)$ th generation. The above-mentioned evolution of a population involves several processes, which are referred to in GA parlance as selection, crossover, mutation and elitism [6].

As suggested by the terminology, the selection process selects two so-called parents from a mating pool consisting of $T$ individuals - where $2 \leq T<P$ - in order to produce two so-called offspring for the next generation population. We shall denote the individuals in the mating pool as $\check{\boldsymbol{b}}_{q}^{(y)}$ for $q=1, \ldots, T$. Two different GA-assisted diversity combining strategies are evaluated here, in order to determine, which $T$ out of the $P$ individuals in a population are placed in the mating pool. A straightforward strategy is to simply sum the two antenna-specific fitness values of each individual in order to produce a diversity-combined fitness value, denoted here as $\Lambda\left(\tilde{\boldsymbol{b}}_{p}^{(y)}\right)=\Lambda_{1}\left(\tilde{\boldsymbol{b}}_{p}^{(y)}\right)+\Lambda_{2}\left(\tilde{\boldsymbol{b}}_{p}^{(y)}\right)$, which was stated explicitly also in Eq. (7), for $p=1, \ldots, P$. Individuals having the $T$ highest diversity-combined fitness values in the population are then placed in the mating pool. Hence our GA-aided optimization employing this strategy is based on the conventional LLF-assisted diversity reception [12]. Intuitively, we can see that this strategy relies more on the exploitation rather than on the exploration of the solution space, since the algorithm always favours individuals having the highest fitness, particularly if $T \ll P$. On the other hand, choosing a higher $T$ value may allow certain low-fitness individuals to be placed in the mating pool and hence may reduce the rate of convergence. The effect of the mating pool size $T$ is investigated in Section IV.

Our second GA-assisted diversity combining strategy is based on the concept of the so-called Pareto optimality [6]. This strategy favours the so-called non-dominated individuals and ignores the so-called dominated individuals. The $p$ th individual is considered to be dominated by the $q$ th individual iff the latter has a higher figure of merit, such as the LLF of Eq. (4), which is formulated as [14]:

$$
\begin{aligned}
& \forall i \in\{1,2\}: \Lambda_{i}\left(\tilde{\boldsymbol{b}}_{q}^{(y)}\right) \geq \Lambda_{i}\left(\tilde{\boldsymbol{b}}_{p}^{(y)}\right) \wedge \\
& \exists i \in\{1,2\}: \Lambda_{j}\left(\tilde{\boldsymbol{b}}_{q}^{(y)}\right)>\Lambda_{j}\left(\tilde{\boldsymbol{b}}_{p}^{(y)}\right) .
\end{aligned}
$$

If an individual is not dominated in the sense of Eq. (8) by any other individuals in the population, by definition it is

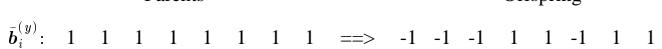

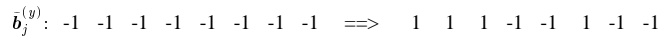

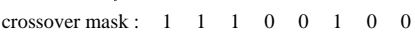

Fig. 2. An example of uniform crossover between two parent bits strings

considered to be non-dominated. According to our second diversity combining strategy, all the non-dominated individuals are placed in the mating pool. Hence the value of $T$ in this case is not fixed, since it depends on the number of non-dominated individuals. Observe that the latter strategy uses the information from both antennas independently, in order to decide which individuals are placed in the mating pool. By contrast, our former strategy based its decisions on a single metric by combining the antennaspecific figures of merit according to Eq. (7).

The individuals in the mating pool are selected as parents according to a probabilistic function based on their corresponding figure of merit $\Lambda\left(\breve{\boldsymbol{b}}_{q}^{(y)}\right)$. In order to prevent premature convergence to a 'local optimum' without exploring the global solution space, the so-called sigma scaling [7] is employed. Under sigma scaling, the selection probability $p\left(\check{\boldsymbol{b}}_{q}^{(y)}\right)$ of an individual is a function of its own fitness as well as that of the mating pool's mean fitness $\bar{\Lambda}$ and its associated standard deviation $\sigma_{\Lambda}$, as formulated below [7] :

$$
p\left(\check{\boldsymbol{b}}_{q}^{(y)}\right)= \begin{cases}1.0+\frac{\Lambda\left(\check{\boldsymbol{b}}_{q}^{(y)}\right)-\bar{\Lambda}}{2 \sigma_{\Lambda}} & \text { if } \sigma_{\Lambda} \neq 0 \\ 1.0 & \text { if } \sigma_{\Lambda}=0\end{cases}
$$

where

$$
\bar{\Lambda}=\frac{1}{T} \sum_{q=1}^{T} \Lambda\left(\check{\boldsymbol{b}}_{q}^{(y)}\right) ; \quad \sigma_{\Lambda}=\sqrt{\frac{\sum_{q=1}^{T}\left[\Lambda\left(\check{\boldsymbol{b}}_{q}^{(y)}\right)-\bar{\Lambda}\right]^{2}}{T-1}} .
$$

The antipodal bits of the parent vectors are then exchanged using the so-called uniform crossover [15] process with a probability $p_{c}$ in order to produce two offspring. Specifically, uniform crossover invokes a so-called crossover mask, which is a sequence consisting of $K$ randomly generated 1 s and 0s. Antipodal bits are exchanged between the pair of parents at locations corresponding to a 1 in the crossover mask. An illustration of the uniform crossover process is shown in Fig. 2. The selection of parents from the mating pool is repeated until a new population of $P$ offspring is produced in order to perform the crossover process.

The mutation process refers to the alteration of the value of an antipodal bit in the offspring from 1 to -1 or vice versa, with a probability $p_{m}$. Finally, under elitism [7], we identify the lowest-merit offspring in the population and replace it with the highest-merit individual from the mating pool. This will ensure that the highest-merit individual is propagated throughout the evolution process.

The GA terminates after $Y$ number of generations. The individual corresponding to the highest scalar fitness value 


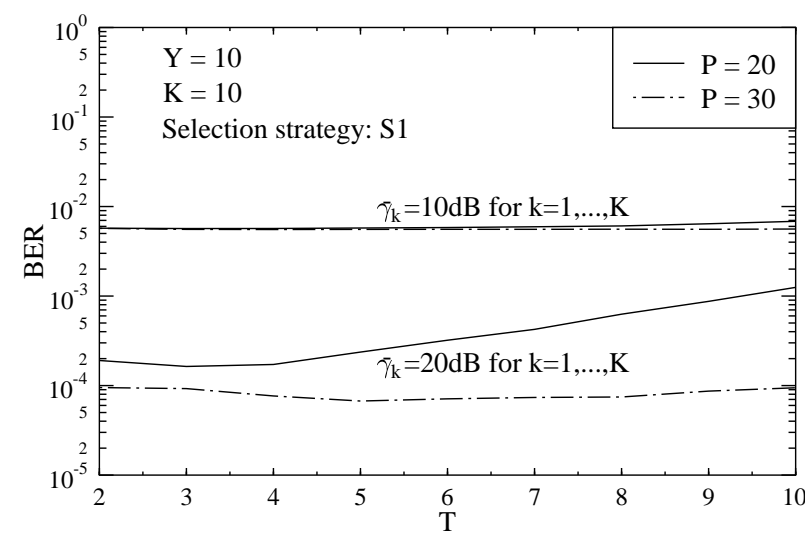

Fig. 3. The BER performance of the GA-based multiuser detector using selection strategy $\mathrm{S} 1$ as a function of the mating pool size $T$ with population sizes $P=20,30$ using binary random signature sequences of length 31 at $\bar{\gamma}_{k}=10,20 \mathrm{~dB}$ for $k=1,2, \ldots, K$. The GA parameters used are the probability of crossover given by $p_{c}=1$, the probability of mutation specified by $p_{m}=0.1$ and the evolution was terminated after $Y=10$ generations

is the detected $K$ number of users' bit sequence associated with the bit-interval considered. For a given population size $P$, the number of LLF evaluations required to detect $\hat{\boldsymbol{b}}$ is equal to $P \times Y \times 2$ for a twin-antenna based diversity scheme. Hence the complexity of the proposed detector is not directly related to the number of users. The values of $P$ and $Y$ can be adaptively selected, in order to find a trade-off between computational complexity and optimum performance.

\section{Simulation Results}

In this section our simulation results are presented, in order to characterize the Bit Error Rate (BER) performance of the GA-based multiuser detector employing the above two GA-based diversity selection strategies highlighted in the previous section. The strategy based on the sum of the figures of merit from both antennas is denoted as S1, while the strategy based on the Pareto optimality is denoted as S2. All the results in this section were based on evaluating the BER performance of a bit-synchronous 10-user CDMA system employing twin-antenna based diversity reception over uncorrelated slowly Rayleigh fading channels. The processing gain was $N_{c}=31$ and the signature sequences were randomly generated.

Fig. 3 shows the effects of the mating pool size $T$ on the BER performance using the selection strategy S1. While the effects of the pool size were negligible at an SNR of $\bar{\gamma}_{k}=10 \mathrm{~dB}$, the deterioration of the BER performance upon increasing $T$ is significant at $\bar{\gamma}_{k}=20 \mathrm{~dB}$ and $P=20$. Furthermore, we can see that the value of $T$ required for achieving the best BER performance was different for diffierent values of $P$, although the dependence on $T$ was not pronounced. According to Fig. $3, T=3$ and $T=5$ gave the best results for $P=20$ and $P=30$, respectively. We will use this result for our subsequent performance studies.

Fig. 4 shows the BER performance against the SNR $\bar{\gamma}_{k}$

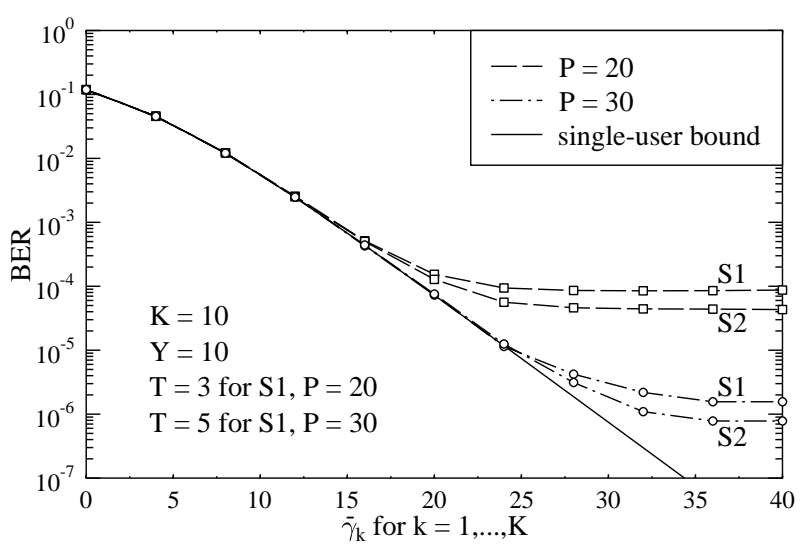

Fig. 4. The BER performance of the GA-based multiuser detector employing the selection strategies of $\mathrm{S} 1$ and $\mathrm{S} 2$ with population sizes of $P=20,30$ using binary random signature sequences of length 31 and supporting $K=10$ users. The average received energy at the antennas was assumed to be equal, i.e. $E\left[\alpha_{k, i}^{2}\right]=0.5$ for $i=1,2$ and $k=1, \ldots, K$. The GA parameters used are the probability of crossover given by $p_{c}=1$, the probability of mutation specified by $p_{m}=0.1$ and the evolution was terminated after $Y=10$ generations

for the GA-based $K=10$-user detector employing selection strategy S1 and S2 with equal average received energy at the two antennas, i.e. for $E\left[\alpha_{k, 1}^{2}\right]=E\left[\alpha_{k, 2}^{2}\right]=0.5$. Perfect power control and CIR estimation was assumed. The single user bound, which assumed equal average received energy at both antennas, was computed using the following equation [13] :

$$
P_{2}=\left[\frac{1}{2}(1-\mu)\right]^{2}(2+\mu)
$$

where $\mu=\sqrt{\frac{\bar{\gamma}_{k}}{1+\bar{\gamma}_{k}}}$. An error floor is observed for the results shown in the figure. As mentioned in Section I, GAs do not always find the optimal solution. In this multiuser scenario it was the GA that caused the error floor and not the multiuser interference. It is seen in Fig. 4 that the BER performance improved, when the population size was increased from $P=20$ to $P=30$. However, this also increased the computational complexity. Hence the values of $P$ can be selected, in order to find a trade-off between computational complexity and performance. We also see from Fig. 4 that the GA employing S2 performs better, exhibiting a lower error floor, as compared to employing S1. Nevertheless, both strategies were capable of matching the single-user bound performance up to $\bar{\gamma}_{k}=16 \mathrm{~dB}$ and $\bar{\gamma}_{k}=24 \mathrm{~dB}$ for $P=20$ and $P=30$, respectively.

We then investigated the BER performance of the GAbased multiuser detector employing selection strategy S1 and S2 with unequal average received energy at the two antennas, setting $E\left[\alpha_{k, 1}^{2}\right]=0.8$ and $E\left[\alpha_{k, 2}^{2}\right]=0.2$. Perfect power control and CIR estimation was assumed again. The results were shown in Fig. 5 in comparison to the singleuser bound given by Eq. (11). Again, we can see that GAs invoking strategy S2 exhibit a lower BER compared 


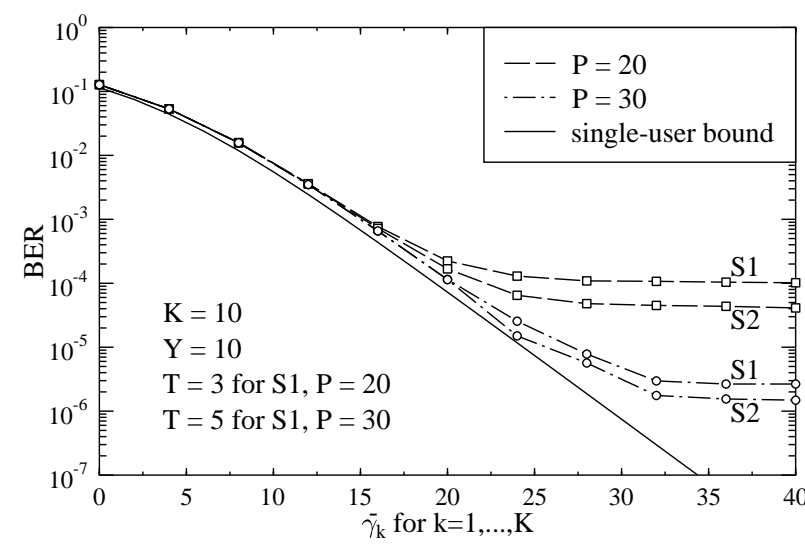

Fig. 5. The BER performance of the GA-based multiuser detector employing selection strategies S1 and S2 with population sizes of $P=20,30$ using binary random signature sequences of length 31 and supporting $K=10$ users. The average received energy at the antennas was assumed to be unequal with $E\left[\alpha_{k, 1}^{2}\right]=0.8$ and $E\left[\alpha_{k, 2}^{2}\right]=0.2$. The GA parameters used are the probability of crossover given by $p_{c}=1$, the probability of mutation specified by $p_{m}=0.1$ and the evolution was terminated after $Y=10$ generations

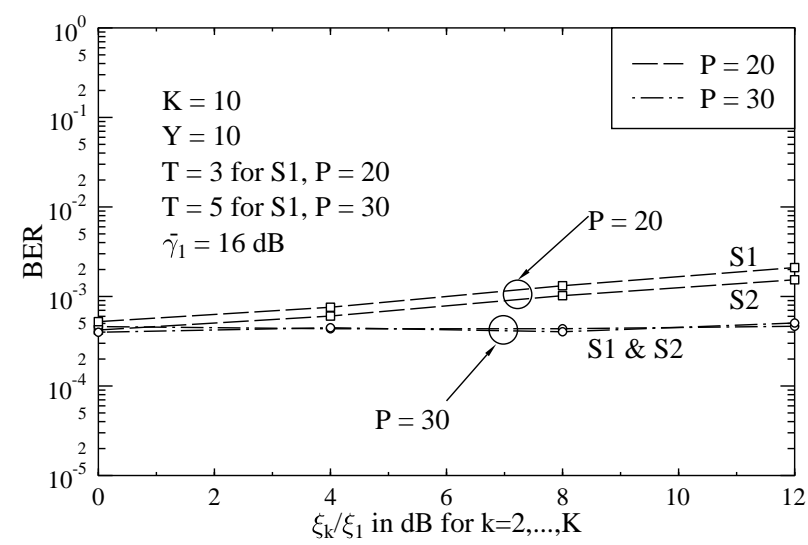

Fig. 6. Relative near-far resistence of the GA-based detector employing selection strategies S1 and S2 with population sizes $P=20,30$ using binary random signature sequences of length 31 at $\bar{\gamma}_{1}=16 \mathrm{~dB}$ and supporting $K=10$ users. The average received energy at the antennas were assumed to be equal. The GA parameters used are the probability of crossover given by $p_{c}=1$, the probability of mutation specified by $p_{m}=0.1$ and the evolution was terminated after $Y=10$ generations

to strategy S1.

Finally, the near-far resistence of the GA-based multiuser detector is demonstrated in Fig. 6 for the desired user. The average energy-to-noise ratio of the desired user $\bar{\gamma}_{1}$ is set to $16 \mathrm{~dB}$, while the energies of all other users were varied in the range of $0-12 \mathrm{~dB}$ higher, than that of the desired user. Perfect CIR estimation was assumed and the average received energy at both antennas were similar. We can see that at population size $P=20$, the BER performance deteriorates slightly, as the interfering users' energy becomes higher relative to the desired user. On the other hand, the BER performance associated with $P=30$ remains almost the same, even when the interfering users' energy is $10 \mathrm{~dB}$ higher than that of the reference user.

\section{Conclusions}

In conclusion, we developed a suboptimal multiuser detector based on GAs in order to circumvent the computational complexity problem present in the optimum multiuser detector [4]. To mitigate the effects of fading, dual antenna diversity techniques were used. Two diversity selection strategies were highlighted for the GAs. In our first solution the mating pool was formed based on the combination of the statistics derived from the diversity antennas and we had a fixed mating pool size. According to our second strategy, the statistics were treated independently, in order to select the non-dominated individuals to form the mating pool. Hence, the mating pool size was not fixed. We have shown that GAs employing the latter strategy always exhibit a lower BER compared to those employing the former strategy. We have also shown that the BER performance can be improved by increasing the population size. The GA-based detector is also near-far resistent. Our future work will attempt to extend these advances to adaptive beam-steering assisted asynchronous CDMA systems, as well as to invoking space-time coding.

\section{REFERENCES}

[1] A. J. Viterbi, "CDMA: Principles of Spread-Spectrum Communications," Addison-Wesley Publishing Company, 1995.

[2] R. Prasad, "CDMA for Wireless Personal Communications," Artech House, Inc., 1996.

[3] S. Verdú, "Multiuser Detection," Cambridge University Press, 1998.

[4] S. Verdú, "Minimum Probability of Error for Asynchronous Gaussian Multiple-Access Channels," IEEE Trans. Inform. Theory, vol. IT-32, no. 1, pp. 85-96, Jan. 1986.

[5] S. Moshavi, "Multi-User Detection for DS-CDMA Communications," IEEE Commun. Mag., vol. 34, no. 10, pp. 124-136, Oct. 1996.

[6] D. E. Goldberg, "Genetic Algorithms in Search, Optimization, and Machine Learning," Addison-Wesley, 1989.

[7] M. Mitchell, "An Introduction to Genetic Algorithms," Cambridge, MA: MIT Press, 1996.

[8] M. J. Juntti, T. Schlosser and J. O. Lilleberg, "Genetic Algorithms for Multiuser Detection in Synchronous CDMA," IEEE Int. Symp. Information Theory - ISIT'97, pp. 492, 1997.

[9] X. F. Wang, W. S. Lu and A. Antoniou, "A Genetic AlgorithmBased Multiuser Detector for Multiple-Access Communications," IEEE Int. Symp. Circuits and System - ISCAS'98, vol. 4, pp. $534-537,1998$.

[10] K. Yen and L. Hanzo, "Hybrid Genetic Algorithm Based Multiuser Detection Schemes for Synchronous CDMA Systems," To appear in VTC'2000 Spring, Tokyo, Japan.

[11] A. Klein, "Known and Novel Diversity Approaches as a Powerful Means to Enhance the Performance of Cellular Mobile Radio Systems," IEEE J. Select. Areas Commun., vol. 14, no. 9, pp. 1784-1795, Dec. 1996.

[12] U. Fawer and B. Aazhang, "A Multiuser Receiver for Code Division Multiple Access Communications over Multipath Channels," IEEE Trans. Commun., vol. 43, no. 2/3/4, pp. 1556-1565, Feb./Mar./Apr. 1995.

[13] J. G. Proakis, "Digital Communications," New York: McGrawHill, 1995.

[14] E. Zitzler and L. Thiele, "Multiobjective Evolutionary Algorithms: A Comparative Case Study and the Strength Pareto Approach," IEEE Tran. Evolutionary Computation, vol. 3, no. 4, pp. 257-271, Nov. 1999.

[15] G. Syswerda, "Uniform Crossover in Genetic Algorithms," Proceedings of the Third International Conference on Genetic Algorithms, Schaffer, J. D. (Ed.), San Mateo, CA: Morgan Kaufmann, pp. 2-9, 1989. 